\title{
Three-Dimensional Nanoscale Chemical Imaging via EFTEM Spectral Imaging
}

\author{
I.M. Anderson, ${ }^{*}$ J.H.J. Scott,* and Z.H. Levine* \\ *National Institute of Standards and Technology, 100 Bureau Dr., Gaithersburg MD 20899-8371
}

Electron scattering methods provide numerous advantages for the three-dimensional (3D) chemical imaging of nanostructures. Amongst particle scattering methods, electrons are uniquely suited to the imaging of individual nanostructures because their strong Coulomb interaction provides short characteristic mean free paths with matter (nanometers) relative to those of X-rays (micrometers) or neutrons (millimeters), and allows electromagnetic fields to be used as lenses for image formation. In comparison with mass spectrometry based techniques, such as atom probe field ion microscopy, electron scattering methods allow the specimen to be interrogated multiple times, provide a wide field of view, and are broadly applicable to metals, alloys, polymers, and biological specimens.

Given the variety of available electron scatting techniques, let us examine some key design criteria for 3D nanoscale chemical imaging in the electron microscope. Electron-transparent specimens are required to achieve the requisite spatial resolution, and the detected signal must be spectroscopic and characteristic, in order to distinguish among chemically distinct phases. Since conventional tomography requires the acquisition of images at numerous projections, any one projection must be acquired in minutes for the data to be acquired "in one sitting." For example, a data set with 90 images $\left(0^{\circ}-180^{\circ}\right.$ in $2^{\circ}$ increments) will take $1.5 \mathrm{~h}$ to acquire for every minute spent on an individual projection (including set-up, acquisition, and data storage). Image quality is also a high priority, since a large number of pixels is desired to fully define the object and image distortions due to the technical aspects of data acquisition will propagate into the $3 \mathrm{D}$ reconstruction and therefore must be avoided. These design criteria align well with the key strengths of energy-filtered transmission electron microscopy (EFTEM), an image-based technique that features rapid data collection and a large number of pixels. A major drawback of EFTEM for tomography is the prevalence of diffraction effects in the analysis of crystalline specimens, which complicates the projection requirement for quantitative tomography [1], that the acquired signal can be transformed by some robust means into a unique scalar variable proportional to the integrated thickness of the phase of interest. Another limitation is nonlinearity due to multiple scattering effects, which alter the spectral intensity distribution for thicker specimens. These effects can be mitigated through spectral imaging methods [2], which allow in particular the deconvolution of multiple scattering effects [3].

3D EFTEM spectral imaging has been applied to a coherent two-phase mixture of face-centered cubic (FCC) transition metal oxides [4,5]. Spectral images were acquired at $0.5^{\circ}$ tilt increments from $-20^{\circ}-+30^{\circ}$ with a Philips CM300FEG operated at $300 \mathrm{kV}$ and equipped with a Gatan Imaging Filter (GIF), $\sim 1 \mathrm{nA}$ total current, $2 \mathrm{~nm}$ pixel size, and $256 \times 256$ pixels. A series of 24 images was acquired with $8 \mathrm{eV} \leq \mathrm{E} \leq 100 \mathrm{eV}, 4 \mathrm{eV}$ increment, $5 \mathrm{eV}$ energy window, and $1 \mathrm{~s}$ exposure (90s total acquisition). Data analysis was performed with the AXSIA spectral image analysis program in PCA image mode with statistical normalization in both spectral and image dimensions [6]. As-acquired images that exhibit a relatively high intensity for the (a) $\mathrm{Ni}_{2} \mathrm{TiO}_{4}$ and (b) $\mathrm{NiO}$ phases are shown in Fig. 1. Although individual images exhibit low contrast, the series of images provides good discrimination between the $\mathrm{Ni}_{2} \mathrm{TiO}_{4}$ (green) and $\mathrm{NiO}$ (blue) phases, as shown in Fig. 2a. A separate spectral component (red) is identified at higher thicknesses because of the redistribution of image intensity due to multiple scattering [6]. A limited subset of the acquired data, comprising images at specimen tilts from $-20^{\circ}-+10^{\circ}$ in 5 increments, is shown in Fig. 2. In order to avoid strong diffracting conditions, the data were acquired with a double-tilt / rotate holder, rotating the specimen along the [020] direction with respect to the FCC lattice so as to maintain weak beam conditions (4g and $5 \mathrm{~g}$ equally excited) along the $[200]_{\mathrm{NiO}}$ systematic row of reflections. However, significant diffraction effects are encountered at zone axes such as [001], at $-12.3^{\circ}$, midway between Figs. $2 \mathrm{~b}, \mathrm{c}$. Ongoing efforts to mitigate diffraction effects and to extend the spectral range will be discussed. 
[1] M. Weyland and P.A. Midgley, Microsc. Microanal. 9 (2003) 542.

[2] I.M. Anderson, Microsc. Microanal. 6 (Suppl. 2) (2000) 1048.

[3] P.J. Thomas and P.A. Midgley, Ultramicrosc. 88 (2001) 187.

[4] I.M. Anderson, C.B. Carter, and H. Schmalzried, Phil. Mag. Lett. 79 (1999) 901.

[5] I.M. Anderson et al., these proceedings.

[6] P.G. Kotula, M.R. Keenan, and J.R. Michael, Microsc. Microanal. 9 (2003) 1.

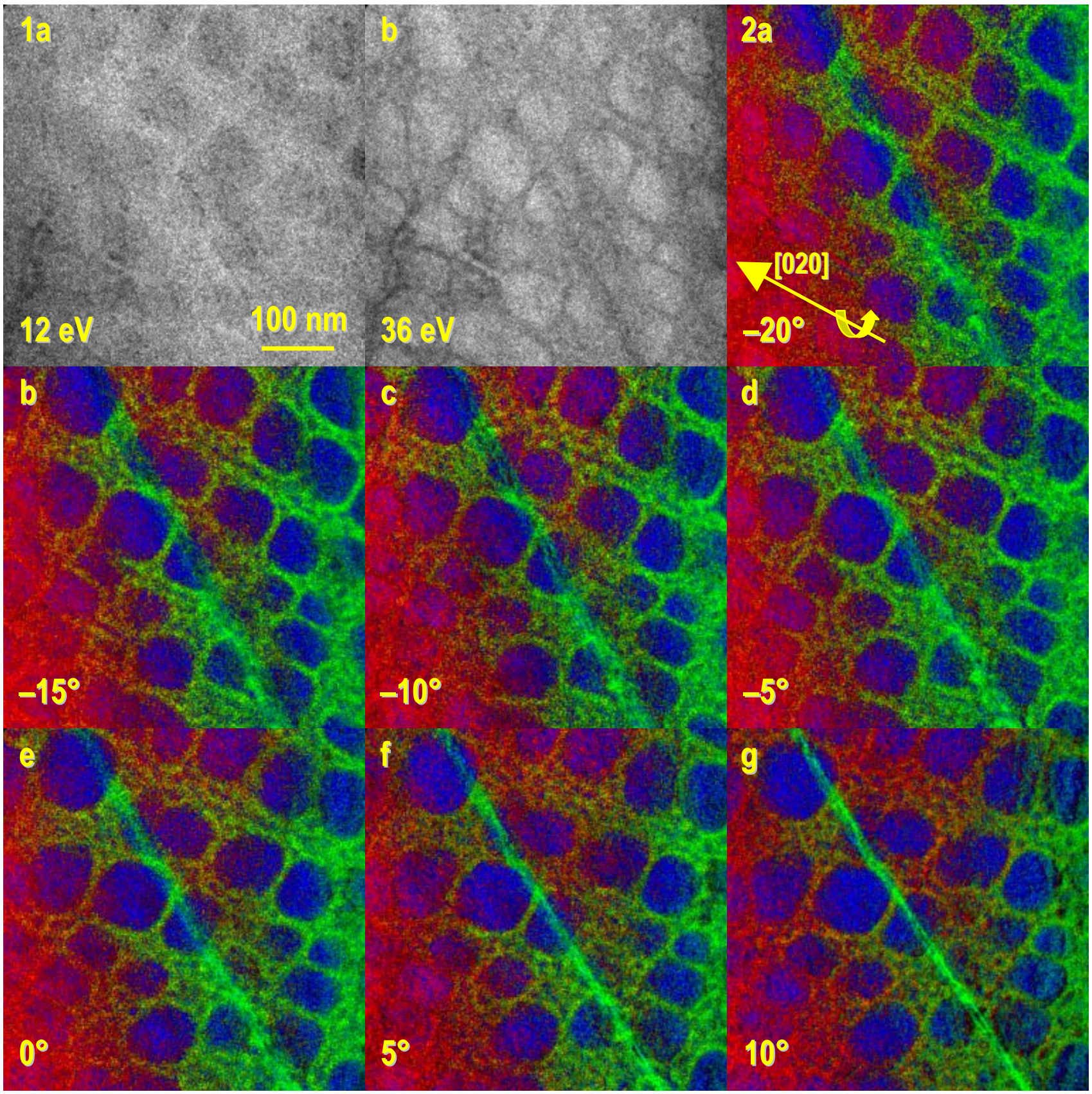

FIG. 1. As-acquired EFTEM images showing higher relative intensity for (a) $\mathrm{Ni}_{2} \mathrm{TiO}_{4}$ and (b) $\mathrm{NiO}$. FIG. 2. RGB reconstructions of EFTEM spectral, acquired at $5^{\circ}$ increments about tilt axis parallel to [020], as indicated in (a). Images (b) and (c) are relatively equally spaced about the [001] zone axis. 